\title{
Electrode Polarization Effects in Broadband Dielectric Spectroscopy
}

\author{
S. Emmert, M. Wolf, R. Gulich, S. Krohns, S. Kastner, P. Lunkenheimer, A. Loidl \\ Experimental Physics V, Center for Electronic Correlations and Magnetism, \\ University of Augsburg, 86135 Augsburg, Germany
}

\begin{abstract}
In the present work, we provide broadband dielectric spectra showing strong electrode polarization effects for various materials, belonging to very different material classes. This includes both ionic and electronic conductors as, e.g., salt solutions, ionic liquids, human blood, and colossal-dielectricconstant materials. These data are intended to provide a broad data base enabling a critical test of the validity of phenomenological and microscopic models for electrode polarization. In the present work, the results are analyzed using a simple phenomenological equivalent-circuit description, involving a distributed parallel RC circuit element for the modeling of the weakly conducting regions close to the electrodes. Excellent fits of the experimental data are achieved in this way, demonstrating the universal applicability of this approach. In the investigated ionically conducting materials, we find the universal appearance of a second dispersion region due to electrode polarization, which is only revealed if measuring down to sufficiently low frequencies. This indicates the presence of a second charge-transport process in ionic conductors with blocking electrodes.
\end{abstract}

\section{INTRODUCTION}

Electrode polarization (EP) leading to blocking electrodes (BEs) is a frequently encountered phenomenon when investigating ionic conductors with impedance or dielectric spectroscopy. This effect, giving rise to giant values of the dielectric constant and a strong drop of conductivity towards low frequencies, arises when the ions arrive at the metallic electrodes and accumulate in thin layers immediately beneath the sample surface forming a so-called space-charge region. It occurs in solid-state electrolytes as well as in aqueous solutions, ionic liquids, and in many biological systems (e.g., [1-18]). EP in ionic conductors often hampers the determination of the intrinsic dielectric properties but it also is of high technical relevance, e.g., in the design of fuel cells or double layer capacitors.

EP also plays an important role in the dielectric spectroscopy of electronic conductors (e.g., [19 31]). In semiconducting samples it is caused, e.g., by the formation of Schottky diodes at the electrode-semiconductor interface. In this case the blocking of the electrode arises from the depletion layer at the semiconductor/metal contact in the blocked state of the diode. Recently these effects came into the focus of interest due to their presumed importance in the so-called "colossal dielectric constant" (CDC) materials [24, 25, 27, 28, 30 32].

The many suggestions made to account for electrode effects in dielectric measurements can be classified into two categories, namely, (i.) avoiding the electrode polarization by specialized measurement techniques or (ii.) modeling these contributions, e.g., by equivalent circuits. The first approach, using, for example, four-electrode methods or varying the electrode distances (e.g., [1, 33, 34]), quite generally implies considerable additional experimental efforts compared to standard dielectric measurements. Thus, often the second approach is adopted and numerous, partly quite sophisticated phenomenological and microscopic models have been proposed to model EP effects in dielectric spectra (e.g., 3 - 7, 12 -16, 24, 35
43]). In dielectric studies, one often deals with situations where the detected EP effects are not in the focus of interest but still cannot be ignored since they have to be taken into account for an unequivocal determination of the intrinsic properties. In such cases, for a straightforward modeling of EP effects, simple equivalent circuits are usually employed, which are assumed to be connected in series to the intrinsic bulk contribution of the sample material. The most common ones are a parallel RC circuit [16, 19, 22, 23, 44, 45] or a so-called constant phase element (CPE) [4, 6, 7, 10, 35, 39, 46], the latter being mainly applied for modeling ionic conductors. Both models work relatively well if only the onset of EP effects is seen, arising close to the low-frequency boundary of the covered frequency range. However, in our experience both approaches have certain drawbacks when analyzing broadband dielectric spectra, where contributions from EP are observed over several frequency decades. Instead we found that a distributed RC equivalent circuit (referred to as DRC circuit henceforth), based on a ColeCole (CC) distribution of relaxation times 21, 47 50], is best suited to describe EP. This is true for such different materials as aqueous solutions, biological systems, solidstate electrolytes, ionic liquids and melts, and all kinds of electronic conductors.

The purpose of the present work is twofold: i.) To provide broadband experimental spectra with strong EP effects in a variety of very different materials that may be helpful for future tests of models of EP (for this purpose, the experimental data are provided in electronic form in the supplementary information. ii.) To demonstrate the universal applicability of the DRC circuit for the modeling of EP effects.

\section{MATERIALS AND METHODS}

Low-frequency measurements up to $\nu \approx 3 \mathrm{MHz}$ were performed using frequency-response analyzers (Novocontrol Alpha-A and Schlumberger 1260 combined with the 
Chelsea dielectrics interface) and an autobalance bridge (Hewlett-Packard HP4284A). Here the sample always was placed in a parallel-plate capacitor. Data in the higher frequency ranges were collected using a coaxial reflection technique, employing the Agilent Impedance/Material Analyzer E4991A (1 MHz - $3 \mathrm{GHz})$ and the autobalance bridge $4294 \mathrm{~A}(40 \mathrm{~Hz}-110 \mathrm{MHz})$. Partly, additional measurements beyond $3 \mathrm{GHz}$ were performed via an open-end coaxial reflection technique and a coaxial transmission technique using the network analyzers Hewlett-Packard 8510 and Agilent E8363B. Further details on the experimental techniques can be found in refs. [8, 18, 51, 52]. Cooling and heating of the samples were achieved by a $\mathrm{N}_{2}$-gas cryostat (Novocontrol Quatro) and various home-made ovens.

In the present publication we provide broadband dielectric data for the following samples (all concentrations in mol percent):

i.) $\mathrm{LiBr} /$ water and $\mathrm{LiBr} /$ glycerol solutions with the concentrations $c=0.018 \%$ and $c=0.73 \%$, respectively. For the preparation of the solutions, $54 \mathrm{wt} \%$ aqueous LiBr solution (Sigma Aldrich), Glycerol (AnalaR Normapur, 99.5\%), $\mathrm{H}_{2} \mathrm{O}$ (Merck "Ultrapur"), and $\mathrm{LiBr}$ powder (Sigma Aldrich, purity 99.999\%) were used.

ii.) $\mathrm{LiCl} /$ water solution with $c=17 \%$ (Sigma Aldrich, purity $>99 \%$ ). [18.

iii.) Human whole blood (hematocrit value of 0.39 )

iv.) An aqueous protein solution: lysozyme with $c=$ 0.009\% (5 mmol/l) (lysozyme: Sigma Aldrich (Fluka)).

v.) The glass-forming ionic melt $\left[\mathrm{Ca}\left(\mathrm{NO}_{3}\right)_{2}\right]_{0.4}\left[\mathrm{KNO}_{3}\right]_{0.6} \quad(\mathrm{CKN})$ (see $\quad$ ref. $[8]$ for preparation details).

vi.) The ionic liquid 1-butyl-3-methylimidazoliumbis(trifluoromethylsulfonyl)imide (BMIM-BMSF) (Iolitec, purity 99\%)

vii.) Single crystalline $\mathrm{La}_{2} \mathrm{CuO}_{4}$, a parent compound of high- $T_{c}$ superconductors (see refs. 19, 21] for details).

viii.) Single crystalline $\mathrm{La}_{15 / 8} \mathrm{Sr}_{1 / 8} \mathrm{NiO}_{4}$, a chargeordered CDC material [53]. [31].

ix.) Polycrystalline $\mathrm{Pr}_{2 / 3} \mathrm{Cu}_{3} \mathrm{Ti}_{4} \mathrm{O}_{12}$, a CDC material

\section{EQUIVALENT-CIRCUIT MODELS FOR ELECTRODE POLARIZATION}

The CPE is often employed to account for EP in ionic conductors 6, 7, 10, 35, 39, 46. Its admittance is defined by $Y^{*}=A(i \nu)^{n}$ with an exponent $n<1$ and the prefactor $A$. A theoretical rationale for the CPE can be obtained when considering a fractional geometry of the electrode/sample interface [4, 5]. A more intuitive modeling of EP effects is provided by a parallel connection of a resistance $R_{\mathrm{E}}$ and a capacitance $C_{\mathrm{E}}$, connected in series to the bulk impedance $Z_{\mathrm{B}}[16,19,22,, 23,44,45]$. Here $C_{\mathrm{E}}$ models the high capacitance of the thin blocking layers and $R_{\mathrm{E}}$ accounts for their high resistance $\left(R_{\mathrm{E}}=\infty\right.$ for complete blocking). This equivalent circuit leads to a total impedance of

$$
Z_{\text {total }}=Z_{\mathrm{B}}+\frac{R_{\mathrm{E}}}{1+i \omega \tau_{\mathrm{E}}}
$$

with $\tau_{\mathrm{E}}=R_{\mathrm{E}} C_{\mathrm{E}}$ and $\omega=2 \pi \nu$ the circular frequency. Assuming, e.g., a frequency-independent bulk resistance and capacitance (i.e., another RC circuit) for the bulk response, this results in so-called Maxwell-Wagner relaxation. It leads to a frequency dependence of the total complex capacitance (and thus of the permittivity $\left.\varepsilon^{*}=\varepsilon^{\prime}-i \varepsilon^{\prime \prime}\right)$ that is fully equivalent to the Debye relaxation law [54] describing the relaxational response of an ideal dipolar system [32, 55]:

$$
\varepsilon^{*}=\varepsilon_{\infty}+\frac{\Delta \varepsilon}{1+i \omega \tau}-i \frac{\sigma_{d c}}{\omega \varepsilon_{0}}
$$

$\tau$ denotes the relaxation time (not identical with $\tau_{\mathrm{E}}[32$ ) and $\Delta \varepsilon=\varepsilon_{\mathrm{s}}-\varepsilon_{\infty}$ the dielectric strength. $\varepsilon_{\mathrm{s}}$ and $\varepsilon_{\infty}$ are the limiting values of the real part of the permittivity for frequencies well below and above the relaxation frequency $\nu_{\text {relax }}=1 /(2 \pi \tau)$, respectively. The last term in eq. (2) was included to account for the contribution of dc charge transport to the dielectric loss $\varepsilon^{\prime \prime} \propto \sigma^{\prime} / \nu$, with $\sigma_{d c}$ the dc conductivity. Typical spectra of $\varepsilon^{\prime}$ and the conductivity $\sigma^{\prime}$ arising from the Maxwell-Wagner relaxation mechanism, described above, are schematically shown by the solid lines in Figs. 1(a) and (b), respectively. It should be noted that in case of the validity of eq. (1), an intrinsic dipolar relaxation and a MaxwellWagner relaxation caused by EP cannot be distinguished based on the measured spectra alone.

However, we found that in many cases the above approach does not lead to a satisfactory description of the experimental data, especially if the spectra extend to frequencies sufficiently low to reveal more than just the onset of the EP effects. Then the spectral features are often found to be more smeared out than expected for this simple equivalent circuit. Interestingly, intrinsic relaxations, e.g., in dipolar glass forming liquids [51], usually exhibit a similar behavior, i.e. a broadening of the observed spectral features. In these cases, the use of an empirical extension of the Debye function, the HavriliakNegami function [56], is well established to account for the observed deviations from Debye behavior:

$$
\varepsilon^{*}(\nu)=\varepsilon_{\infty}+\frac{\Delta \varepsilon}{\left[1+(i \omega \tau)^{1-\alpha}\right]^{\beta}}-i \frac{\sigma_{d c}}{\omega \varepsilon_{0}}
$$

The above function with $0 \leq \alpha<1$ and $0<\beta \leq 1$ includes the special cases with $\beta=1$ and $\alpha=0$, termed Cole-Cole [57] and Cole-Davidson [58, 59] function, respectively. These two additional parameters lead to a broadening of the spectral features, which is commonly ascribed to a distribution of relaxation times. While 


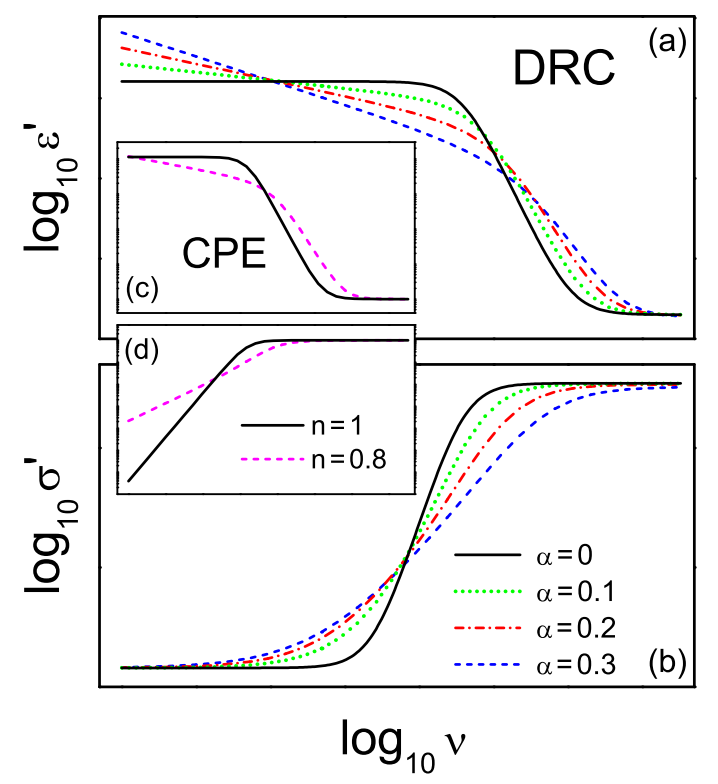

FIG. 1: (a) Dielectric constant and (b) real part of the conductivity calculated according to the "Cole-Cole"-case $(\beta=1)$ of eq. (4) for various values of the distribution parameter $\alpha$. $\alpha=0$ corresponds to the undistributed case, eq. (11). For the bulk, frequency independent $\epsilon^{\prime}$ and $\sigma^{\prime}$ were assumed, i.e. a simple parallel $\mathrm{RC}$ circuit connected in series to the impedance of eq. 4. Frames (c) and (d) show corresponding curves for a CPE with $n=1$ and $n=0.8$ instead of a DRC element.

the Havriliak-Negami and the Cole-Davidson functions are purely empirical, the Cole-Cole distribution of $\tau$ can be approximated by the microscopic model of Gaussdistributed energy barriers [60]. This formula leads to a symmetric broadening (compared to the Debye case) of the relaxation peak in $\varepsilon^{\prime \prime}$ and, e.g., is often used for the description of secondary relaxations of glassy matter [61, 62]. However, we found that a direct application of eq. (3) to Maxwell-Wagner relaxations arising from EP cannot account for the experimental data. An alternative approach is an extension of eq. (11) analogous to eq. (3), arriving at

$$
Z_{\text {total }}=Z_{\mathrm{B}}+\frac{R_{\mathrm{E}}}{\left[1+\left(i \omega \tau_{\mathrm{E}}\right)^{1-\alpha}\right]^{\beta}}
$$

with $0 \leq \alpha<1,0<\beta \leq 1$. For the Cole-Cole case $(\beta=1)$, this equation was already considered earlier $47-$ [50]. A generalized Havriliak-Negami version as in eq. (4) is also included in the "LEVM" fit programm by Prof. J. Ross Macdonald [63]. However, until now this DRC equivalent circuit has only rarely been used for the modeling of EP effects [47, 48, 64]. As shown in the further course of the present work, it seems that the $\mathrm{CC}$ version of eq. (4) provides good fits of EP-dominated spectra in a large variety of very different materials.

It has to be stressed that, whereas a simple RC equivalent circuit (eq. (11)), connected in series to the bulk, produces permittivity curves identical to a Debye function (eq. (2)), a DRC equivalent circuit based on eq. (41) in general produces permittivity spectra that are qualitatively different from those of a Havriliak-Negami function, eq. (3). For the latter, the relaxation time $\tau$ in Eq. (3) is assumed to be distributed. In the equivalent-circuit case, the corresponding quantity is $\tau=R_{B} C_{\mathrm{E}}\left(R_{B}\right.$ denotes the bulk resistance), which determines, e.g., the loss peak position via $\nu_{p}=1 /(2 \pi \tau)$, just like $\tau$ in eq. (3) 24, 32]. However, the quantity that is distributed in the equivalent-circuit case is $\tau_{E}=R_{\mathrm{E}} C_{\mathrm{E}}$, thus leading to different curve shapes.

Finally it should be mentioned that the DRC circuit for the Cole-Cole case and a parallel connection of a CPE and a resistor can produce identical frequency dependences of the dielectric quantities [50]. The DRC circuit seems to be the more physical one because fitting the experimental data with this model provides direct access to the resistances and capacitances of the blocking surface layers. However, as will become clear in the further course of this work, the extended CPE element may find an explanation in a second, slower hopping transport process within the BE layers [15].

Figure 1 shows typical spectra of $\varepsilon^{\prime}(\mathrm{a})$ and $\sigma^{\prime}$ (b) calculated by assuming a DRC equivalent circuit to account for the EP. For comparison, spectra using a plain RC circuit (solid lines in (a) and (b)) and a CPE (frames (c) and (d)), instead of the DRC, are provided. In all cases for the bulk response a simple parallel RC circuit was assumed, corresponding to a frequency independent bulk $\varepsilon^{\prime}$ and $\sigma^{\prime}$. For the distribution, the CC case (i.e., $\beta=1$ and $\alpha \neq 0$ in eq. (4) was chosen. The curves for the undistributed RC circuit (solid lines), which are identical to Debye behavior, eq. (21), show the typical dispersion steps in $\varepsilon^{\prime}$ and $\sigma^{\prime}$. With increasing $\alpha$, these steps become smeared out for both quantities. In addition, the low-frequency plateau of $\varepsilon^{\prime}(\nu)$ develops into a weak power law. The low and high-frequency plateaus of $\sigma^{\prime}(\nu)$ remain unaffected (however, we found that for very high values of $\alpha$ the $\sigma^{\prime}(\nu)$ curve can cross those limits). Comparing this behavior with that of the CPE case (Fig. 1(c) and (d)), the most obvious difference is the missing low-frequency plateau in $\sigma^{\prime}(\nu)$ for the latter (d). This would correspond to completely blocking electrodes. However, adding a parallel resistance to the CPE leads to such a plateau and, as mentioned above, in this case both circuits produce identical curves.

In the following, several experimental spectra being governed by strong EP effects, obtained on partly very different materials, will be fitted by the models introduced above. All fits were simultaneously performed for $\varepsilon^{\prime}(\nu)$ and $\sigma^{\prime}(\nu)$. For the experimental data, statistical errors were assumed. 


\section{RESULTS AND DISCUSSION}

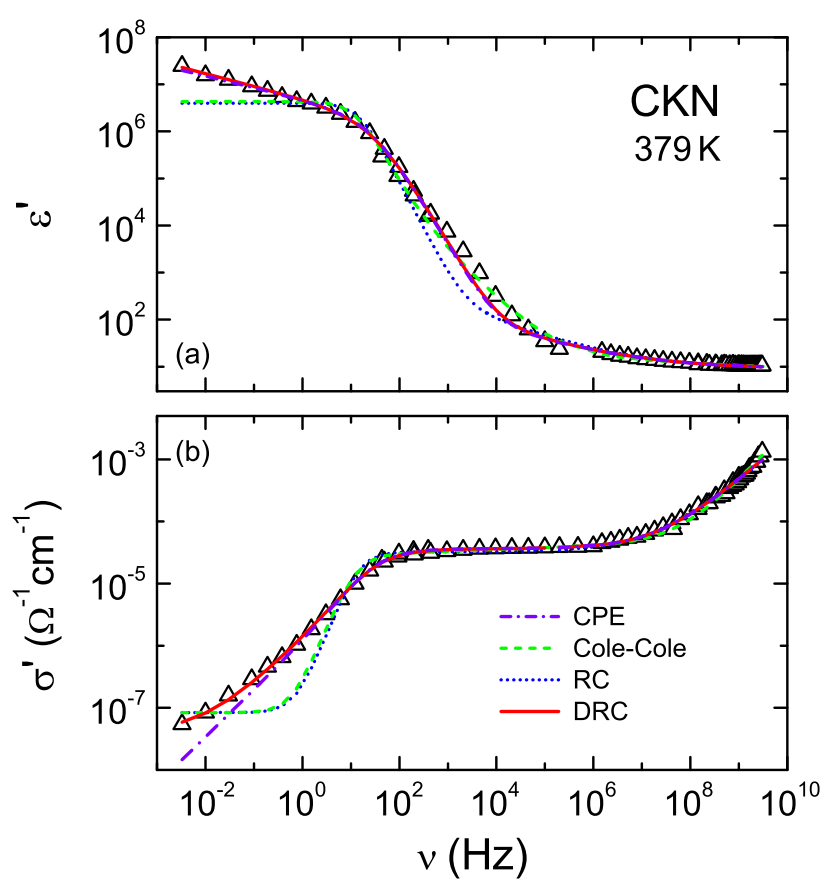

FIG. 2: (a) Dielectric constant and (b) real part of the conductivity of CKN at $379 \mathrm{~K}$ as function of frequency. The lines represent fits using different ways to account for the EP effects: equivalent circuits with a CPE (dash-dotted lines), a parallel RC circuit, eq. (1) (dotted lines), or a DRC circuit, eq. (4) with $\beta=1$ (solid lines), connected in series to the bulk. For the latter, a power-law increase in $\sigma^{\prime}(\nu)$ and the corresponding contribution in $\varepsilon^{\prime}(\nu)$ was used [55]. For comparison, the dashed lines show fit curves where the EP effect was treated like an intrinsic relaxation and fitted by a ColeCole function plus dc-conductivity contribution (eq. (3) with $\beta=1$ ).

Figure 2 shows broadband spectra of $\varepsilon^{\prime}(\nu)$ and $\sigma^{\prime}(\nu)$ of $\mathrm{CKN}$ at $379 \mathrm{~K}$, extending over 12 frequency decades [8, 65]. In this supercooled ionic melt, EP effects lead to a strong increase of the dielectric constant and a strong decrease of the conductivity at low frequencies. The increase of $\sigma^{\prime}(\nu)$ at high frequencies is of intrinsic nature and corresponds to an onset of a high-frequency minimum in the dielectric loss, $\varepsilon^{\prime \prime} \propto \sigma^{\prime} / \nu$. This typical feature of glass formers is treated in detail in [65, [66], where data extending to even higher frequencies are provided. The dotted and dash-dotted lines in Fig. 2 show fits with the two standard descriptions of EP, namely a parallel RC circuit and a CPE, respectively, both connected in series to the bulk. For the bulk, in addition to the usual parallel RC circuit, we have assumed a power-law increase in $\sigma^{\prime}(\nu)$ and the corresponding contribution in $\varepsilon^{\prime}(\nu)$ to phenomenologically account for the mentioned high-frequency effects [55]. The EP-generated low-frequency dispersions in $\varepsilon^{\prime}(\nu)$ and $\sigma^{\prime}(\nu)$ of CKN are clearly too smeared out to be fitted by a simple RC circuit (dotted line). In addition, the absence of a clear low-frequency plateau in $\varepsilon^{\prime}(\nu)$ is not taken into account by this approach. Applying this RC equivalent-circuit is mathematically equivalent to fitting the data with an intrinsic Debye relaxation, eq. (22). Thus one may expect better fits if using a broadened function like the Cole-Cole function, eq. (3) with $\beta=1$. However, as demonstrated by the dashed lines in Fig. 2, such fits are still unsatisfactory. This is also the case for the Havriliak-Negami and Cole-Davidson variant of eq. (3) (not shown).

While the CPE (dash-dotted lines in Fig. 2) does a good job in the description of $\varepsilon^{\prime}(\nu)$, it fails to account for the onset of a low-frequency plateau observed in $\sigma^{\prime}(\nu)$ at $\nu<0.1 \mathrm{~Hz}$. This plateau, which is often overlooked because of a limited frequency range or low ion mobility, obviously is due to an incomplete blocking of the electrodes. It indicates a second conductivity mechanism that starts to prevail at $\nu<10^{-1} \mathrm{~Hz}$ when the main charge-transport mechanism is blocked. It could be due to electronic conductivity or to a second ion species with much lower mobility, which is not yet blocked at the lowest frequencies investigated [14]. However, it seems unlikely that the mobilities of the different ion species $\left(\mathrm{K}^{+}\right.$, $\mathrm{Ca}^{2+}$, and $\mathrm{NO}_{3}^{-}$in the present case) differ by about three orders of magnitude as suggested by a comparison of the plateaus at $\nu \rightarrow 0$ and $\nu \approx 10^{4} \mathrm{~Hz}$ [67]. Another possibility is that the ions located within the space charge region, close to the electrode, still have some mobility left. In line with the model proposed in ref. [15], this mobility can be assumed to be much lower than in the bulk, but not zero, thus explaining the approach of an additional low-frequency plateau in $\sigma^{\prime}(\nu)$. Then it seems plausible that at extremely low frequencies a second, final blocking effect should arise, i.e. $\sigma^{\prime}(\nu)$ should decrease again when the ions within the space charge region finally reach the sample boundary. As shown in the further course of this work, this is indeed observed in several of the investigated materials.

As demonstrated by the solid lines in Fig. 2, modeling the BEs by a DRC circuit yields satisfying fitting curves of $\varepsilon^{\prime}(\nu)$ and $\sigma^{\prime}(\nu)$ in the whole frequency range, including the onset of a low-frequency plateau in the conductivity. The resistance arising from the second chargetransport mechanism is taken into account by the resistor within the parallel $\mathrm{RC}$ equivalent circuit, modeling the EP effects. We find $\alpha=0.26$ and $\beta=1$, indicating a moderate, symmetrical distribution of relaxation times $\tau_{E}$ (Cole-Cole case). Broadband spectra obtained at other temperatures in CKN [8, 65] also can be well modeled in this way. In fact, in an earlier work [64] a sophisticated model that included a DRC circuit (termed "ZC" there) to account for EP, was used to obtain excellent fits of spectra in CKN at four temperatures between 342 and $361 \mathrm{~K}$.

Figure 3 provides results on BMIM-BMSF, an ionic liq- 

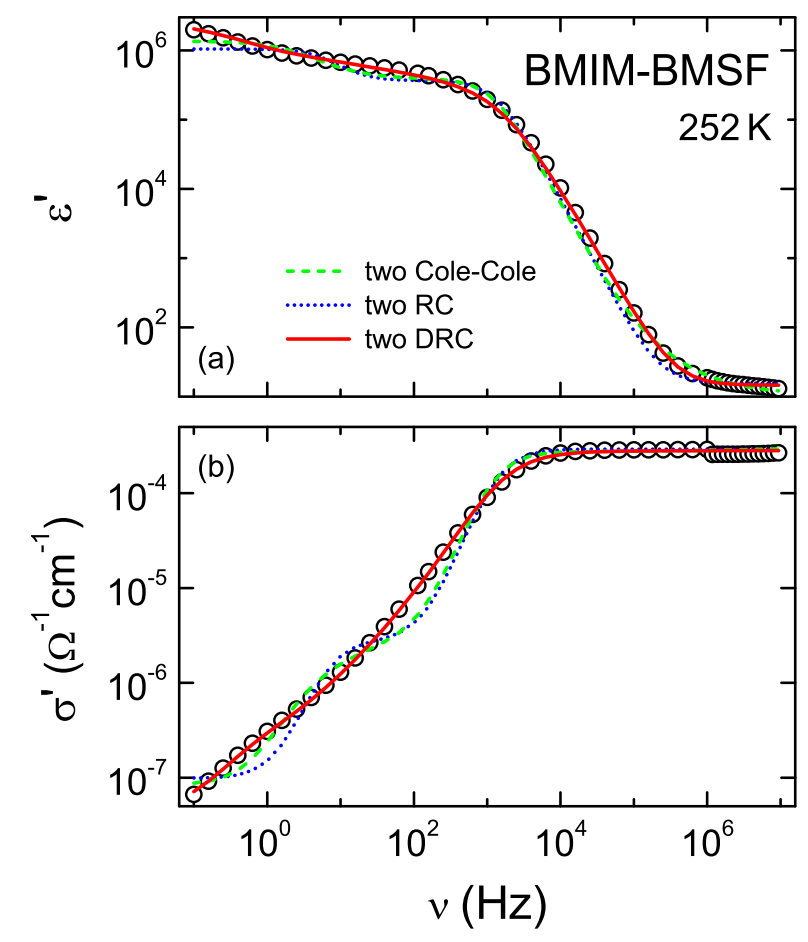

FIG. 3: (a) Dielectric constant and (b) real part of the conductivity of BMIM-BMSF at $252 \mathrm{~K}$ as function of frequency. The lines represent fits assuming different models for the EP effects: the sum of two Cole-Cole functions, eq. (3) with $\beta=1$ (dashed lines), two parallel RC circuits, eq. (1), connected in series to the bulk (dotted lines), and two DRC circuits, eq. (4) with $\beta=1$, also connected in series to the bulk impedance (solid lines). For the latter two cases, the bulk is modeled by a parallel RC circuit.

uid, i.e. a substance solely consisting of cations and anions that is liquid at room temperature. This class of materials has attracted considerable interest in recent years due to their possible technical relevance as environmentfriendly solvents and as new types of electrolytes used, e.g., in batteries [68, 69]. Again, typical EP effects are observed in $\varepsilon^{\prime}(\nu)(\mathrm{a})$ and $\sigma^{\prime}(\nu)(\mathrm{b})$. The upward bending of the $\varepsilon^{\prime}(\nu)$ curve in the upper-plateau region of the main dispersion step below $\nu \approx 10 \mathrm{~Hz}$ clearly indicates the presence of a second dispersion step. In Fig. 3(b), after the initial decrease of $\sigma^{\prime}(\nu)$ below about $3 \times 10^{3} \mathrm{~Hz}$, one may suspect the approach of a second plateau at around $10 \mathrm{~Hz}$ with amplitude of $\sim 10^{-6} \Omega^{-1} \mathrm{~cm}^{-1}$, which is followed by a second decrease below about $1 \mathrm{~Hz}$. As discussed above, the latter finding may well indicate the final blocking of a second charge-transport mechanism. Admittedly, the mentioned features are not well pronounced and rather smeared out. However, we found that fits with a single DRC element are not able to provide a good description of the present data. Instead, the experimental spectra can be very well fitted by assuming two DRC elements in series to the bulk (solid lines) modeling the blocking of the two transport mechanisms. Here the bulk is assumed to have frequency-independent $\varepsilon^{\prime}$ and $\sigma^{\prime}$, i.e. it is modeled by another (undistributed) parallel RC circuit. From the fits, we obtain $\alpha=0.18$ and $\beta=1$ for the DRC element, modeling the main dispersion step. For the low-frequency one, a pure RC element $(\alpha=0, \beta=1)$ was sufficient, which may be due to the fact that it is only partly visible in the investigated frequency range. As indicated by the dotted lines in Fig. 3, a combination of two undistributed RC circuits (eq. (1)) in series to the bulk, cannot explain the observed frequency dependence. Using instead the sum of two Cole-Cole functions (eq. (3) with $\beta=1$; dashed lines), i.e. treating the data as arising from two intrinsic dipolar relaxations with different time constants, also does not account for the experimental data.

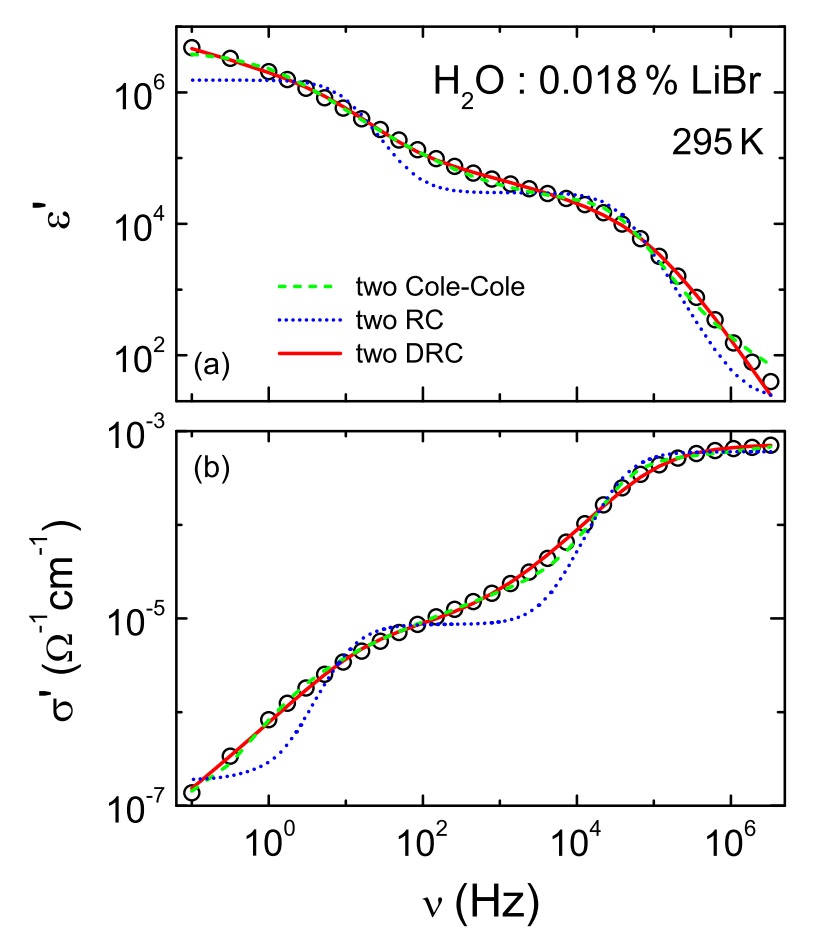

FIG. 4: (a) Dielectric constant and (b) conductivity of a $0.018 \% \mathrm{LiBr} /$ water solution at $295 \mathrm{~K}$ as function of frequency. The meanings of the lines are the same as in Fig. 3 .

As an example for a classical electrolyte solution, Fig. 4 provides the frequency dependence of $\varepsilon^{\prime}$ and $\sigma^{\prime}$ of a $0.018 \% \mathrm{LiBr} /$ water solution at $295 \mathrm{~K}$. The real part of the permittivity (a) reveals two smeared-out dispersion steps with points of inflection at around 10 and $10^{5} \mathrm{~Hz}$, which are caused by EP effects. The conductivity (b) also shows two dispersion features. The high-frequency plateau in $\sigma^{\prime}(\nu)$ of about $10^{-3} \Omega^{-1} \mathrm{~cm}^{-1}$ corresponds to 
the intrinsic ion conductivity of this solution, dominated by the main charge-transport process (see discussion of the CKN data). This system exhibits a high conductivity and an onset of the EP effects at rather high frequencies (about $10^{5} \mathrm{~Hz}$ in $\sigma^{\prime}(\nu)$, compared to $3 \times 10^{3} \mathrm{~Hz}$ in BMIMBMSF at $252 \mathrm{~K}$ and $10^{2} \mathrm{~Hz}$ in CKN at $379 \mathrm{~K}$, cf. Figs. 3 and 2). Probably for this reason, the appearance of a second plateau (at about $10^{2} \mathrm{~Hz}$ and $\sim 10^{-5} \Omega^{-1} \mathrm{~cm}^{-1}$ ) is well pronounced in this electrolyte and, in addition, the final blocking is well seen (further decrease of $\sigma^{\prime}(\nu)$ below $\sim 10 \mathrm{~Hz}$ ). Both effects clearly indicate a second conductivity mechanism that becomes blocked at lower frequencies, as already suspected in the discussion of the CKN and BMIM-BMSF data.

The lines in Fig. 4 again are fits with different empirical functions to account for the BEs. As demonstrated by the dotted lines, two parallel RC equivalent circuits (eq. (10), both connected in series to the bulk impedance, obviously cannot account for the experimental data. Here again the bulk was assumed to have frequency-independent $\varepsilon^{\prime}$ and $\sigma^{\prime}$. The sum of two ColeCole functions (eq. (3) with $\beta=1$ ) leads to much better, but still not perfect fits (dashed lines). In contrast, assuming two DRC elements, eq. (4), with $\beta=1$, connected in series to the bulk (solid line), provides nearly perfect fits. The values of the distribution parameter $\alpha$ are 0.32 (for the low frequency EP effect) and 0.28 (for the high frequency EP effect).

As an example for the temperature-dependent development of EP effects, Fig. 5 shows $\varepsilon^{\prime}(\nu)$ (a) and $\sigma^{\prime}(\nu)$ (b) of a $0.73 \% \mathrm{LiBr} /$ glycerol solution for various temperatures between $220 \mathrm{~K}$ and $410 \mathrm{~K}$. For the lowest temperature shown $(220 \mathrm{~K})$ only the intrinsic $\alpha$-relaxation of glycerol (cooperative reorientation of the dipolar glycerol molecules [51]) and the bulk conductivity arising from the ionic charge transport can be seen in this frequency range. This relaxation is characterized by steps in $\varepsilon^{\prime}(\nu)$ and in $\sigma^{\prime}(\nu)$ (the latter corresponding to the wellknown loss peaks in $\varepsilon^{\prime \prime}(\nu)$ [51]). The bulk conductivity leads to the low-frequency plateau in $\sigma^{\prime}(\nu)$ of about $10^{-10} \Omega^{-1} \mathrm{~cm}^{-1}$. Due to the low ion mobility at this temperature, the EP effects lie outside the investigated frequency range. With increasing temperature, the mobility rises and the EP shows up as a strong increase in $\varepsilon^{\prime}(\nu)$ at low frequencies that finally develops into a steplike curve. In addition, a decrease in the conductivity for decreasing frequency arises above $240 \mathrm{~K}$. At even higher temperatures, a second, smeared-out step is revealed in $\varepsilon^{\prime}(\nu)$, accompanied by a weak corresponding feature in $\sigma^{\prime}(\nu)$. Similar to the behavior in the aqueous $\mathrm{LiBr}$ solution (Fig. 4), this further dispersion indicates a second charge-transport mechanism as discussed above. As demonstrated by the lines in Fig. 5 perfect fits of all these spectra are achieved by assuming a DRC circuit (eq. (4)) with $\beta=1$ for the description of the observed EP effects, connected in series to the bulk response. For the latter, the $\alpha$-relaxation is accounted for by the ColeDavidson function, eq. (3) with $\alpha=0$ [51, 70]. The
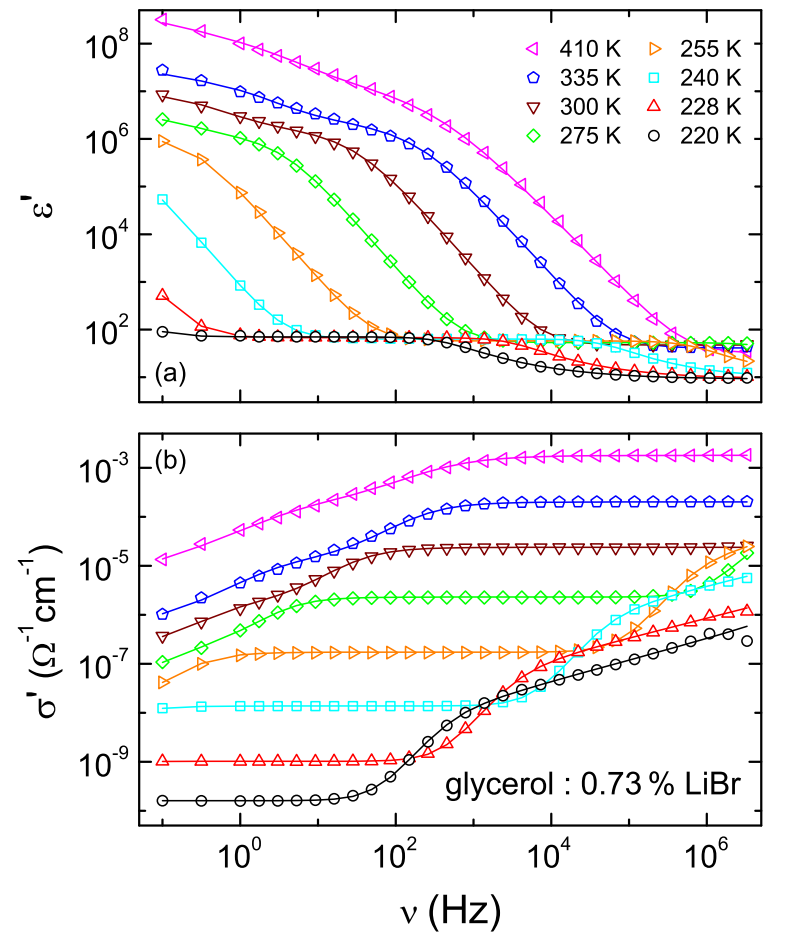

FIG. 5: (a) Dielectric constant and (b) conductivity of a $0.73 \% \mathrm{LiBr} /$ glycerol solution at different temperatures as function of frequency. The lines are fits with two DRC circuits, eq. (4) with $\beta=1$, in series to the bulk to account for the EP. For the bulk, an intrinsic dipolar relaxation, described by the Cole-Davidson formula and a frequency-independent dc conductivity were used (eq. (3) with $\alpha=0$ ).

distribution parameters $\alpha$ of the slower EP effect vary between 0.1 and 0.33 ; for the second one we obtained values between 0.1 and 0.29 .

Further examples of dielectric spectra, dominated by EP are provided in Fig. 6. It shows the dielectric properties of a variety of partly very different materials, including a highly concentrated salt solution, two biological samples, and three electronic conductors. The squares represent the results obtained on a $17 \% \mathrm{LiCl}$ /water solution at $290 \mathrm{~K}$. Its frequency spectra reveal two strong dispersion steps in $\varepsilon^{\prime}(\nu)$ (a) and $\sigma^{\prime}(\nu)(\mathrm{b})$. As demonstrated by the lines, the spectra are well fitted with two Cole-Cole DRC elements in series to the sample impedance, which is modeled by a simple RC circuit. The broadening of the low-frequency dispersion step is characterized by $\alpha=0.22$; the faster one could be modeled with a pure RC equivalent circuit (i.e. $\alpha=0, \beta=1$ ).

The triangles in Fig. 6 represent the broadband data of human blood at $290 \mathrm{~K}$ [18]. Blood can be considered as an aqueous solution of a variety of different salts with the addition of a considerable fraction of suspended "particles", mainly red blood cells. As treated in detail in ref. [18], at $\nu>10^{5} \mathrm{~Hz}$, those data are dominated 

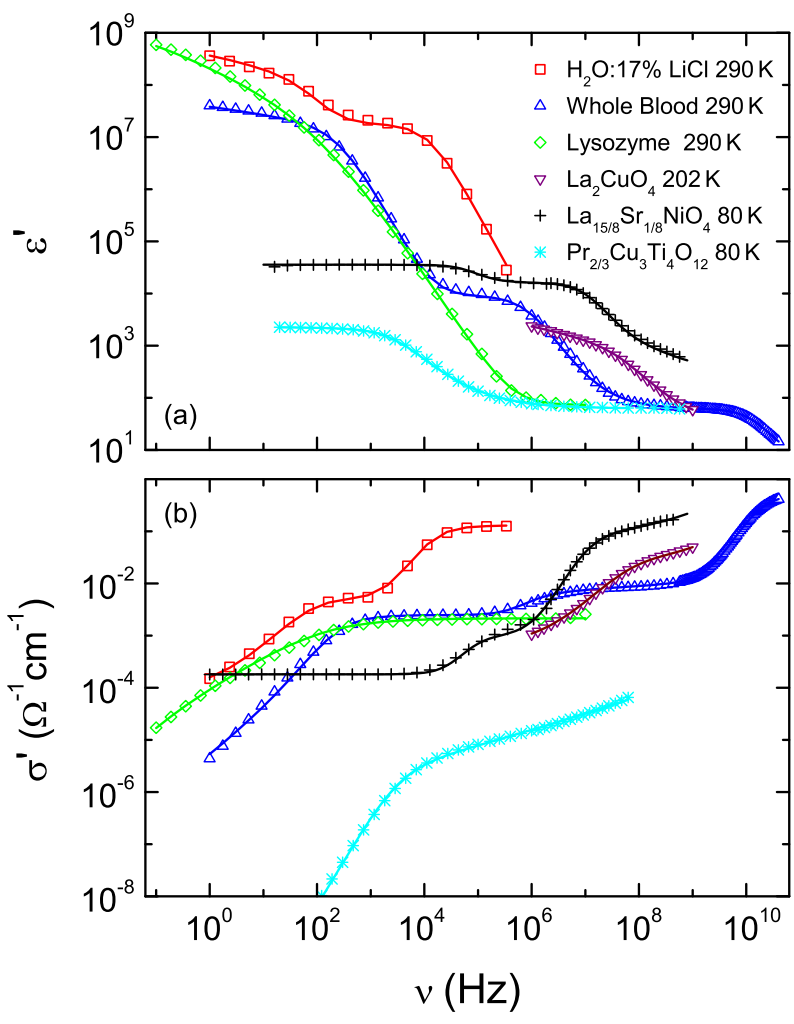

FIG. 6: (a) Dielectric constant and (b) real part of the conductivity of different samples that are affected by EP effects: $17 \% \mathrm{LiCl} /$ water solution at $290 \mathrm{~K}$ (squares), human whole blood at $290 \mathrm{~K}$ (triangles), 0.009\% Lysozym/water solution (diamonds), single-crystalline $\mathrm{La}_{2} \mathrm{CuO}_{4}$ (down triangles), single crystalline $\mathrm{La}_{15 / 8} \mathrm{Sr}_{1 / 8} \mathrm{NiO}_{4}$ (crosses), and polycrystalline $\mathrm{Pr}_{2 / 3} \mathrm{Cu}_{3} \mathrm{Ti}_{4} \mathrm{O}_{12}$ (stars). The lines are fits with one or two DRC circuits, connected in series to the bulk, which is modeled by a parallel RC circuit.

by the well-known $\beta$ - and $\gamma$-dispersions of blood [18, 71]. The $\beta$-dispersion (at about $10^{6}-10^{7} \mathrm{~Hz}$ ) arises from a Maxwell-Wagner relaxation caused by the membrane of the red blood cells 71]. The $\gamma$-dispersion (at about $10^{10} \mathrm{~Hz}$ ) is caused by the cooperative reorientational motion of water molecules [18, 71]. At $\nu<10^{5} \mathrm{~Hz}$, strong EP effects show up in the blood spectra of Fig. 6. The complete broadband spectra are well fitted with a single Cole-Cole DRC circuit (eq. (41) with $\beta=1$ ), connected in series to the bulk impedance. The latter is modeled by the sum of two "normal" Cole-Cole functions (i.e. they are defined in $\varepsilon^{*}$; eq. (3) with $\beta=1$ ) to account for the $\beta$ - and $\gamma$-dispersions. The finding that a single DRC circuit is sufficient to account for the data may be ascribed to the fact that the onset of the EP effects in blood occurs at rather low frequencies, comparable to the results in CKN (Fig. 2).

The diamonds in Fig. 6 show the results for a $0.009 \%$ (5 mmol/l) Lysozyme solution. Protein solutions usu- ally contain free ions and indeed EP effects show up in the present spectra, leading to strong dispersion in both quantities. The very gradual transition to a lowfrequency plateau in $\varepsilon^{\prime}(\nu)$ at $\nu<10^{2} \mathrm{~Hz}$ indicates the presence of a second, smaller step on top of the main dispersion. Indeed, excellent fits of the experimental spectra were achieved by using two DRC elements in series to the bulk. The DRC circuit leading to the smaller, lowfrequency step is Debye-like $(\alpha=0, \beta=1)$; the broad main dispersion step is characterized by $\alpha=0.46$ and $\beta=1$.

Finally, Fig. [6]also provides some typical data on electronically conducting materials, where the EP arises from the formation of Schottky diodes at the electrode/sample interface. Even when the polarity of the oscillating field changes, one of the two oppositely poled diodes at both sides of the sample always is blocked and dominates the response at low frequencies. In recent years, especially EP effects in non-metallic transition-metal oxides have gained increasing significance: Many of these materials show effects like magnetocapacitance, multiferroicity or the occurrence of very large dielectric constants that have triggered numerous dielectric investigations [32, 72, 73]. However, EP effects can considerably hamper the detection of their intrinsic dielectric properties and even be misinterpreted as being of intrinsic origin [24, 25, 74, 75]. The examples provided in Fig. 6 include single-crystalline $\mathrm{La}_{2} \mathrm{CuO}_{4}$, a parent compound of high- $T_{c}$ superconductors [19, 21], single crystalline $\mathrm{La}_{15 / 8} \mathrm{Sr}_{1 / 8} \mathrm{NiO}_{4}$, a charge-ordered CDC material [53], and polycrystalline $\mathrm{Pr}_{2 / 3} \mathrm{Cu}_{3} \mathrm{Ti}_{4} \mathrm{O}_{12}$ [31], a material structurally closely related to the well-known CDC material $\mathrm{CaCu}_{3} \mathrm{Ti}_{4} \mathrm{O}_{12}$ 76]. For all these materials, strong EP effects are revealed in Fig. [6] which look qualitatively similar to those in the ionic conductors. In all cases, the spectra can well be fitted by using a DRC circuit to account for the EP (lines). For $\mathrm{La}_{15 / 8} \mathrm{Sr}_{1 / 8} \mathrm{NiO}_{4}$ a second DRC circuit was used to cover the second dispersion step seen below about $10^{6} \mathrm{~Hz}$. In ref. [53], by measurements with different contact types the high-frequency step was clearly identified to arise from EP. The origin of the low-frequency step is not clarified yet but it was speculated that it may be connected to the inhomogeneous charge distribution in this prototypical charge-ordered material [53]. We also found the DRC to be applicable for the description of EP effects in $\mathrm{Cu}_{2} \mathrm{Ta}_{4} \mathrm{O}_{12}$ 25] and $\mathrm{CaCu}_{3} \mathrm{Ti}_{4} \mathrm{O}_{12}$, another CDC material [77].

\section{SUMMARY AND CONCLUSIONS}

In the present work, we have provided dielectric spectra showing strong EP effects measured in a variety of materials. They partly belong to very different material classes like salt solutions, ionic liquids and melts, biological materials, and electronic semiconductors. Our results are intended to provide a comprehensive data base for comparing current and future approaches for the mod- 
eling of EP effects in dielectric spectra. For this purpose, the data are made available in electronic form in the supplementary information. In addition, in the present work we have demonstrated that the DRC equivalent circuit is an easy-to-apply phenomenological model for fitting EP effects in dielectric spectra, which works well for all the different material classes investigated. However, this model certainly cannot be regarded as an alternative to microscopic models as discussed, e.g., in refs. [12, 14, 15, 41 43]. On the other hand, it has proven useful for a quick, phenomenological description of EP effects, enabling fits of experimental data in the complete investigated frequency range, which can help in the determination of the intrinsic bulk properties of the investigated material.

In all the investigated ionically conducting materials, we find evidence for a second charge-transport process that leads to a second plateau in the frequency-dependent conductivity at frequencies below the commonly observed decrease of $\sigma^{\prime}(\nu)$ due to blocking. In addition, in most of these materials we find a further decrease of $\sigma^{\prime}(\nu)$ at the lowest frequencies investigated, indicating final blocking of the charge carriers involved in this second process. These seemingly quite universal features have been only rarely detected so far: In most works on ionic conductors only the onset of the EP effects is observed in the spectra, and the data can be fitted by simple models like an undistributed RC circuit or a CPE. It is unlikely that the second dispersion, observed in measurements extending to sufficiently low frequencies, is due to a second, much slower ion species or due to electronic conduction. Instead, to us an explanation in line with the model proposed in ref. [15] seems more likely: The same ion species moves fast in the bulk region and much slower in the space-charge region, thus leading to the observed two dispersion effects. In ref. [15], a specific hopping-conductivity model [78] was used to model the transport process in the region close to the electrode. Here we use the DRC circuit with $\beta=1$, which is equivalent to a CPE connected in parallel to a resistor [50]. A CPE, sometimes termed "universal dielectric response" [55], is often employed to describe hopping conductivity in various materials and indeed it leads to very similar frequency dependence as the model considered in [15]. Thus, using the DRC circuit is in line with the model of ref. [15] and adding a second DRC element also accounts for the final blocking of the slow ions in the BE region.

It is an interesting finding that the DRC element also provides a perfect description of the EP effects observed in electronic conductors. In fact, $\mathrm{La}_{2} \mathrm{CuO}_{4}$ (Fig. 6) was the first material, where we introduced this circuit for the description of an electronic conductor 15 years ago [21]. Great care has to be taken to avoid the misinterpretation of relaxation-like features in the spectra of semiconducting materials as intrinsic effects that could be indicative, e.g., of ferroelectricity or "colossal" dielectric constants. Thus the correct modeling of these effects is essential. The fact that the present DRC-circuit approach leads to a different frequency dependence than an intrinsic relaxation process, modeled, e.g., by a Cole-Cole function (see, e.g., Fig. 3), provides some possibility to distinguish between intrinsic and extrinsic effects. In addition, of course the procedures proposed in ref. [24] (e.g., performing measurements with different contact types, etc.) should be performed to arrive at a definite conclusion.

This work was partly supported by the Deutsche Forschungsgemeinschaft via Research Unit FOR 1394 and via the Transregional Collaborative Research Center TRR 80. We thank A. Fälschle for performing the measurements in BMIM-BMSF.
[1] H.P. Schwan, Ann. N.Y. Acad. Sci. 148, 191 (1968)

[2] J.E. Bauerle, J. Phys. Chem. Solids 30, 2657 (1969)

[3] W. Scheider, J. Phys. Chem. 79, 127 (1975)

[4] S.H. Liu, Phys. Rev. Lett. 55, 529 (1985)

[5] L. Nyikos, T. Pajkossy, Electrochim. Acta 30, 1533 (1985)

[6] J.B. Bates, Y.T. Chu, W.T. Stribling, Phys. Rev. Lett. 60, 627 (1988)

[7] J.R. Macdonald, J. Electroanal. Chem. 223, 25 (1987)

[8] A. Pimenov, P. Lunkenheimer, H. Rall, R. Kohlhaas, A. Loidl, R. Böhmer, Phys. Rev. E 54, 676 (1996)

[9] A. Pimenov, J. Ullrich, P. Lunkenheimer, A. Loidl, C.H. Ruscher, Solid State Ionics 109, 111 (1998)

[10] F. Bordi, C. Cametti, T. Gili, Bioelectrochem. 54, 53 (2001)

[11] Y. Feldman, I. Ermolina, Y. Hayashi, IEEE Trans. Dielectr. Electr. Insul. 10, 728 (2003)

[12] M.Z. Bazant, K. Thornton, A. Ajdari, Phys. Rev. E 70, 021506 (2004)
[13] R.J. Klein, S.H. Zhang, S. Dou, B.H. Jones, R.H. Colby, J. Runt, J. Chem. Phys. 124, 144903 (2006)

[14] H. Sanabria, J.H. Miller, Phys. Rev. E 74, 051505 (2006)

[15] A. Serghei, M. Tress, J.R. Sangoro, F. Kremer, Phys. Rev. B 80, 184301 (2009)

[16] J.C. Dyre, P. Maass, B. Roling, D.L. Sidebottom, Rep. Prog. Phys. 72, 046501 (2009)

[17] R. Gulich, M. Köhler, P. Lunkenheimer, A. Loidl, Radiat. Environ. Biophys. 48, 107 (2009)

[18] M. Wolf, R. Gulich, P. Lunkenheimer, A. Loidl, Biochim. Biophys. Acta. in press., arXiv: 1105.5061 (2010)

[19] P. Lunkenheimer, M. Resch, A. Loidl, Y. Hidaka, Phys. Rev. Lett. 69, 498 (1992)

[20] O. Bidault, P. Goux, M. Kchikech, M. Belkaoumi, M. Maglione, Phys. Rev. B 49, 7868 (1994)

[21] P. Lunkenheimer, G. Knebel, A. Pimenov, G.A. Emelchenko, A. Loidl, Z. Phys. B: Condens. Matter 99, 507 (1996)

[22] A. Seeger, P. Lunkenheimer, J. Hemberger, A.A. Mukhin, 
V.Y. Ivanov, A.M. Balbashov, A. Loidl, J. Phys. Condens. Matter 11, 3273 (1999)

[23] A.I. Ritus, A.V. Pronin, A.A. Volkov, P. Lunkenheimer, A. Loidl, A.S. Shcheulin, A.I. Ryskin, Phys. Rev. B 65, 165209 (2002)

[24] P. Lunkenheimer, V. Bobnar, A.V. Pronin, A.I. Ritus, A.A. Volkov, A. Loidl, Phys. Rev. B 22, 052105 (2002)

[25] P. Lunkenheimer, R. Fichtl, S.G. Ebbinghaus, A. Loidl, Phys. Rev. B 70, 172102 (2004)

[26] N. Biskup, A. de Andres, J.L. Martinez, C. Perca, Phys. Rev. B 72, 024115 (2005)

[27] C.C. Wang, L.W. Zhang, Appl. Phys. Lett. 88, 042906 (2006)

[28] G. Deng, T. Yamada, P. Muralt, Appl. Phys. Lett. 91, 202903 (2007)

[29] S. Krohns, P. Lunkenheimer, S.G. Ebbinghaus, A. Loidl, J. Appl. Phys. 103, 084107 (2008)

[30] M.C. Ferrarelli, D.C. Sinclair, A.R. West, H.A. Dabkowska, A. Dabkowski, G.M. Luke, J. Mater. Chem. 19, 5916 (2009)

[31] J. Sebald, S. Krohns, P. Lunkenheimer, S.G. Ebbinghaus, S. Riegg, A. Reller, A. Loidl, Solid State Commun. 150, 857 (2010)

[32] P. Lunkenheimer, S. Krohns, S. Riegg, S.G. Ebbinghaus, A. Reller, A. Loidl, Eur. Phys. J. Special Topics 180, 61 (2010)

[33] M.M. Springer, A. Korteweg, J. Lyklema, J. Electroanal. Chem. 153, 55 (1983)

[34] B.A. Mazzeo, A.J. Flewitt, J. Appl. Phys. 102, 104106 (2007)

[35] H. Fricke, Philos. Mag. 14, 310 (1932), ISSN 1941-5982

[36] H.C. Chang, G. Jaffe, J. Chem. Phys. 20, 1071 (1952)

[37] J.R. Macdonald, Phys. Rev. 92, 4 (1953)

[38] D.R. Franceschetti, J.R. Macdonald, J. Appl. Phys. 50, $291(1979)$

[39] Y. Feldman, R. Nigmatullin, E. Polygalov, J. Texter, Phys. Rev. E 58, 7561 (1998)

[40] J.R. Macdonald, J. Non-Cryst. Solids 307, 913 (2002)

[41] J.R. Macdonald, J. Phys. Condens. Matter 22, 495101 (2010)

[42] J.R. Macdonald, L.R. Evangelista, E.K. Lenzi, B. G., J. Phys. Chem. C 115, 7648 (2011)

[43] M.Z. Bazant, B.D. Storey, A.A. Kornyshev, Phys. Rev. Lett. 106, 046102 (2011)

[44] H.P. Schwan, C.D. Ferris, Rev. Sci. Instrum. 39, 481 (1968)

[45] M. Umino, N. Oda, Y. Yasuhara, Med. Biol. Eng. Comput. 40, 533 (2002)

[46] P.H. Bottelberghs, G.H.J. Broers, J. Electroanal. Chem. 67, 155 (1976), ISSN 0022-0728

[47] D. Ravaine, J.L. Souquet, C.R. Hebd. Seances Acad. Sci. 277, 489 (1973)

[48] J.R. Sandifer, R.P. Buck, J. Electroanal. Chem. 56, 385 (1974)

[49] J.R. Macdonald, in Superionic Conductors, edited by
G.D. Mahan, W.L. Roth (Plenum Press, New York, 1976), p. 81

[50] D.R. Franceschetti, J.R. Macdonald, in Impedance Spectroscopy, edited by E. Barsoukov, J.R. Macdonald (Wiley, Hoboken, 2005), chap. 2, p. 80

[51] P. Lunkenheimer, U. Schneider, R. Brand, A. Loidl, J. Contemp. Phys. 41, 15 (2000)

[52] U. Schneider, P. Lunkenheimer, A. Pimenov, R. Brand, A. Loidl, Ferroelectrics 249, 89 (2001)

[53] S. Krohns, P. Lunkenheimer, C. Kant, A.V. Pronin, H.B. Brom, A.A. Nugroho, M. Diantoro, A. Loidl, Appl. Phys. Lett. 94, 122903 (2009)

[54] P. Debye, Polar Molecules (Dover Publications, Inc., New York, 1929)

[55] A.K. Jonscher, Dielectric Relaxations in Solids (Chelsea Dielectrics Press, London, 1983)

[56] S. Havriliak, S. Negami, J. Polym. Sci. C 14, 99 (1966)

[57] K.S. Cole, R.H. Cole, J. Chem. Phys. 9, 341 (1941)

[58] D.W. Davidson, R.H. Cole, J. Chem. Phys. 18, 1417 (1950)

[59] R.H. Cole, D.W. Davidson, J. Chem. Phys. 20, 1389 (1952)

[60] U.T. Hochli, K. Knorr, A. Loidl, Adv. Phys. 39, 405 (1990)

[61] A. Kudlik, S. Benkhof, T. Blochowicz, C. Tschirwitz, E. Roessler, J. Mol. Struct. 479, 201 (1999)

[62] S. Kastner, M. Köhler, Y. Goncharov, P. Lunkenheimer, A. Loidl, J. Non-Cryst. Solids 357, 510 (2011)

[63] J.R. Macdonald, Homepage (2010), http://www.jrossmacdonald.com/

[64] J.R. Macdonald, J. Phys. Chem. B 111, 7064 (2007)

[65] P. Lunkenheimer, A. Loidl, in Broadband Dielectric Spectroscopy, edited by F. Kremer, A. Schönhals (Springer, Berlin, 2002), chap. 5

[66] P. Lunkenheimer, A. Pimenov, A. Loidl, Phys. Rev. Lett. 78, 2995 (1997)

[67] M.C.C. Ribeiro, Phys. Chem. Chem. Phys. 6, 771 (2004)

[68] T. Welton, Chem. Rev. 99, 2071 (1999)

[69] M. Armand, F. Endres, D.R. MacFarlane, H. Ohno, B. Scrosati, Nat. Mater. 8, 621 (2009)

[70] M. Köhler, P. Lunkenheimer, A. Loidl, Eur. Phys. J. E 27, 115 (2008)

[71] H.P. Schwan, Adv. Biol. Med. Phys. 5, 147 (1957)

[72] N.A. Spaldin, M. Fiebig, Science 309, 391 (2005)

[73] S.W. Cheong, M. Mostovoy, Nat. Mater. 6, 13 (2007)

[74] G. Catalan, Appl. Phys. Lett. 88, 102902 (2006)

[75] A. Loidl, S. Krohns, J. Hemberger, P. Lunkenheimer, J. Phys. Condens. Matter 20, 191001 (2008)

[76] C.C. Homes, T. Vogt, S.M. Shapiro, S. Wakimoto, A.P. Ramirez, Science 293, 673 (2001)

[77] B. Renner, P. Lunkenheimer, M. Schetter, A. Loidl, A. Reller, S.G. Ebbinghaus, J. Appl. Phys. 96, 4400 (2004)

[78] J.C. Dyre, Phys. Lett. A 108, 457 (1985) 